\title{
An audit of the management of elderly patients with glioblastoma in the UK: have recent trial results changed treatment?
}

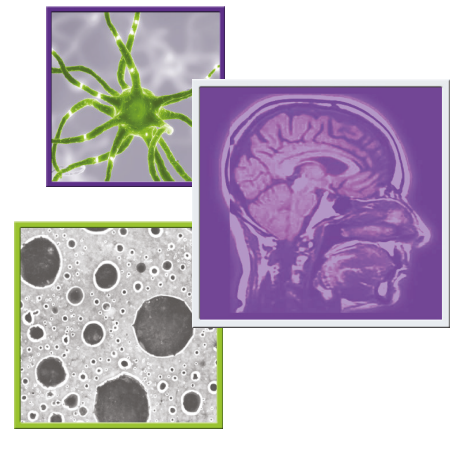
\author{
Catherine McBain ${ }^{3}$, Philip McLoone ${ }^{4}$ \& Anthony J Chalmers ${ }^{5}$ \\ ${ }^{1} \mathrm{NHS}$ Greater Glasgow \& Clyde, Glasgow, Scotland, G51 4TF, UK \\ ${ }^{2}$ Brighton \& Sussex University Hospitals NHS Trust, Brighton, England, BN2 5BB, UK \\ ${ }^{3}$ The Christie NHS Foundation Trust, Manchester, England, M20 4BX, UK \\ ${ }^{4}$ Institute of Health \& Wellbeing, University of Glasgow, Glasgow, Scotland, G12 8RZ, UK \\ ${ }^{5}$ Institute of Cancer Sciences, University of Glasgow, Glasgow, Scotland, G12 OYN, UK \\ *Author for correspondence: m.chong1@nhs.net
}

Ming Yao Chong*,1, Cressida F Lorimer ${ }^{2}$, Shaveta Mehta ${ }^{3}$, Ehab Ibrahim ${ }^{3}$, Juliet Brock ${ }^{2}$,

\begin{abstract}
Aim: We investigated uptake of short-course chemo-radiotherapy and compared outcomes with other treatment schedules in elderly patients with glioblastoma (GBM). Methods: Patients aged 65 or over with a diagnosis of GBM were identified from an 18-month period from three centers in the UK. The primary end point of this study was overall survival from the date of diagnosis. Results: The analysis included 210 patients. Overall median survival was 5.0 months. Approximately $31.9 \%$ of patients received combined chemoradiation; multivariate analysis showed that patients who received standard chemoradiation were at a reduced risk of death than those receiving hypofractionated chemoradiation. Discussion: In this retrospective study, patients treated with standard chemoradiation experienced better outcomes than patients receiving hypofractionated chemoradiation. Patient selection likely contributed to these findings.
\end{abstract}

First draft submitted: 20 September 2019; Accepted for publication: 15 October 2019; Published online: 10 December 2019

Glioblastoma (GBM) is a primary brain tumor that confers a very poor prognosis [1]. Elderly patients (generally defined as age $\geq 65$ years) represent nearly half of the GBM population and have a median survival of 7.2 months compared with 11.2 months in the nonelderly population [2-4].

Standard management of GBM comprises surgical resection followed by standard radiotherapy (60 Gy in 30 fractions) with concomitant and adjuvant temozolomide chemotherapy, as defined by Stupp et al. [5]. While this treatment strategy has improved overall survival for younger patients with GBM, elderly patients were underrepresented in the study and those over 70 were excluded. Subgroup analysis showed no survival benefit from adding chemotherapy to radiotherapy in patients aged 66-70 [6].

Several randomized Phase III trials have addressed uncertainties surrounding the management of GBM in elderly patients [7-9]. Roa et al. [8] compared standard radiotherapy to hypofractionated radiotherapy (40 Gy in 15 fractions) and Wick et al. [7] compared chemotherapy only (temozolomide) to standard radiotherapy, with neither study finding a difference in survival. The most recent study, undertaken by Perry et al. [9], demonstrated that adding concomitant and adjuvant temozolomide to hypofractionated radiotherapy significantly improved survival in elderly patients compared with hypofractionated radiotherapy alone (9.3 vs 7.6 months; hazard ratio for death 0.67; $\mathrm{p}<0.001)$ However, no direct comparisons have yet been made between standard and hypofractionated chemo-radiotherapy, and many clinicians prefer to offer the standard regimen to patients aged 65 and over who have good performance status. With this in mind, we undertook a retrospective study of the management of elderly patients with GBM in three UK centers, to evaluate the use of hypofractionated chemo-radiotherapy and to estimate overall survival in patients receiving different treatment regimens.

\section{Patients \& methods}

This retrospective study was conducted in three UK centers: Beatson West of Scotland Cancer Centre (Glasgow), Christie Hospital (Manchester) and Sussex Cancer Centre (Brighton). These were selected as tertiary cancer care

Future Medicine 
centers with large catchment areas representing diverse UK regions. Patients were identified from neuro-oncology multi-disciplinary team (MDT) meeting lists between 1 July 2016 and 31 December 2017. Inclusion criteria were broad: aged 65 and over; diagnosis of GBM made either by pathology or radiology as confirmed by a consultant neuro-radiologist in an MDT meeting and no involvement in a therapeutic clinical trial. Clinical data were collected retrospectively from electronic patient information databases using MDT notes, clinical letters, operation notes, radiology and pathology reports as primary sources. Follow-up data was collected up to 18 March 2019. As this was an audit, approval by an ethics review committee was not required.

\section{Clinical characteristics}

Baseline information comprised age, sex, Eastern Cooperative Oncology Group (ECOG) performance status (PS) and date of diagnosis, defined either as date of surgery or date of diagnostic radiological imaging if no histological diagnosis was available.

\section{Management}

Chemotherapy and radiotherapy treatment details were recorded, including radiotherapy dose, number of fractions and whether treatment was completed. Standard radiotherapy was defined as $60 \mathrm{~Gy}$ in 30 fractions and hypofractionated radiotherapy as $40 \mathrm{~Gy}$ in 15 fractions. Surgery was classified into gross total resection $(>90 \%$ tumor resection), subtotal resection (<90\% tumor resection) and biopsy.

\section{Pathology}

Pathological diagnosis and tumor grade were based on the WHO classification of 2007. Information on IDH-1 and $M G M T$ promoter methylation status were recorded where available.

\section{Statistical analyses}

Chi-square and Mann-Whitney tests were used to investigate differences in distributions of patient characteristics by treatment group. To visualize overall mortality, Kaplan Meier survival curves were plotted, and log-rank tests were used to evaluate differences between treatment groups. Median survival times were estimated and presented with 95\% CIs. For patients who had received combined chemotherapy and radiotherapy, Cox's proportional hazards regression was used to produce univariate, traditional multivariable adjusted and propensity score adjusted hazard ratios for hypofractionated radiotherapy versus standard radiotherapy. Treatment regimen, sex, age and PS were included as factors which may have had a bearing on patient outcome. To account for imbalances in the distribution of these covariates, propensity scores were calculated using logistic regression, with treatment group (hypofractionated radiotherapy) as the outcome and age, sex, PS and treatment center as predictors of treatment received; adjustments were made by including the resulting propensities when modeling hazard ratios. Median follow-up time was estimated using the reverse Kaplan Meier method. All tests were two-sided, p-values less than 0.05 were considered statistically significant. All analyses were performed using STATA v14.2 (StataCorp, TX, USA).

\section{Results}

Demographics

A total of 217 patients with a diagnosis of GBM and aged over 65 years were identified. Four patients were excluded because of participation in a clinical trial and three were excluded due to insufficient information, leaving 210 patients included in the final analysis. Table 1 shows the patient characteristics. Mean age was 74.2 years (standard deviation 6.29 ) and $56.2 \%$ of patients were males. The most commonly reported PS score was 1 (35\% of patients) and ranged from $0(11 \%)$ to $3+(30 \%)$.

\section{Diagnosis \& genetic testing}

A diagnosis of GBM was made via histopathological examination in 122 patients, and by radiological confirmation by a consultant neuro-radiologist in 88 patients. Tumor specimens from 80 patients (38\%) underwent testing for MGMT promoter methylation status, of which 25 were methylated, 50 were unmethylated and five did not yield a result. Immunohistochemical $I D H-1$ data were available for 108 patients, of whom only one exhibited IDH-1 mutation. Neurosurgery was performed on 126 patients as detailed in Table 1. In total, 30 patients (23.8\%) underwent stereotactic biopsy, 58 (46.0\%) subtotal resection and 37 (29.4\%) gross total resection. 


\begin{tabular}{|c|c|c|c|c|c|}
\hline Patient characteristics & All patients & $\begin{array}{l}\text { Chemotherapy and } \\
\text { radiotherapy }\end{array}$ & Chemotherapy only & Radiotherapy only & Supportive treatment \\
\hline Number & 210 & $67(31.9)$ & $16(7.6)$ & $23(11.0)$ & $104(49.5)$ \\
\hline Mean age (SD) & $74.2(6.29)$ & $69.8(4.0)$ & $72.5(6.1)$ & $72.5(6.0)$ & $76.8(6.0)$ \\
\hline \multicolumn{6}{|l|}{ Age group (years): } \\
\hline$-65-<70$ & $71(33.8)$ & $40(59.7)$ & $7(43.8)$ & $10(43.5)$ & $14(13.5)$ \\
\hline$-70-<75$ & $50(23.8)$ & $18(26.9)$ & $2(12.5)$ & $6(26.1)$ & $24(23.1)$ \\
\hline$-75+$ & $89(42.4)$ & $9(13.4)$ & $7(43.8)$ & $7(30.4)$ & $66(63.5)$ \\
\hline \multicolumn{6}{|l|}{ Sex: } \\
\hline - Men & $118(56.2)$ & $35(52.2)$ & $8(50.0)$ & $14(60.9)$ & $61(58.7)$ \\
\hline - Women & $92(43.8)$ & $32(47.8)$ & $8(50.0)$ & $9(39.1)$ & $43(41.3)$ \\
\hline \multicolumn{6}{|l|}{ Performance status: } \\
\hline-0 & $23(11.0)$ & $17(25.4)$ & $1(6.3)$ & $2(8.7)$ & $3(2.9)$ \\
\hline-1 & $73(34.8)$ & $31(46.3)$ & $12(75.0)$ & $9(39.1)$ & $21(20.2)$ \\
\hline-2 & $41(19.5)$ & $9(13.4)$ & $2(12.5)$ & $7(30.4)$ & $23(22.1)$ \\
\hline$-3+$ & $61(29.0)$ & $5(7.5)$ & $1(6.3)$ & $3(13.0)$ & $52(50.0)$ \\
\hline - Unknown & $12(5.7)$ & $5(7.5)$ & $0(0.0)$ & $2(8.7)$ & $5(4.8)$ \\
\hline \multicolumn{6}{|l|}{ Treatment center: } \\
\hline - Glasgow & $100(47.6)$ & $16(23.9)$ & $16(100.0)$ & $3(13.0)$ & $65(62.5)$ \\
\hline - Brighton & $37(17.6)$ & $9(13.4)$ & $0(0.0)$ & $6(26.1)$ & $22(21.2)$ \\
\hline - Manchester & $73(34.8)$ & $42(62.7)$ & $0(0.0)$ & $14(60.9)$ & $17(16.3)$ \\
\hline \multicolumn{6}{|l|}{ MGMT promoter methylation: } \\
\hline - Tested & 80 & 32 & 16 & 9 & 21 \\
\hline - Methylated & $25(31.3)$ & $9(28.1)$ & $10(62.5)$ & $2(22.2)$ & $4(19.0)$ \\
\hline - Unmethylated & $50(62.5)$ & $23(71.9)$ & $6(37.5)$ & $6(66.7)$ & $15(71.4)$ \\
\hline - Failed testing & $5(6.3)$ & $2(6.3)$ & $0(0.0)$ & $1(11.1)$ & $2(9.5)$ \\
\hline \multicolumn{6}{|l|}{ Neurosurgery: } \\
\hline - Stereotactic biopsy & $30(14.3)$ & $7(10.4)$ & $7(43.8)$ & $6(26.1)$ & $10(9.6)$ \\
\hline - Subtotal resection $<90 \%$ & $58(27.6)$ & $42(62.7)$ & $4(25.0)$ & $4(17.4)$ & $8(7.7)$ \\
\hline - Gross total resection $>90 \%$ & $37(17.6)$ & $18(26.9)$ & $4(25.0)$ & $6(26.1)$ & $9(8.7)$ \\
\hline - Unknown surgery & $1(4.8)$ & $0(0.0)$ & $1(6.3)$ & $0(0.0)$ & $0(0.0)$ \\
\hline - No surgery & $84(40.0)$ & $0(0.0)$ & $0(0.0)$ & $7(30.4)$ & $77(74.0)$ \\
\hline \multicolumn{6}{|l|}{ Diagnosis: } \\
\hline - Pathological diagnosis & $122(58.1)$ & $67(100.0)$ & $16(100.0)$ & $16(69.6)$ & $23(22.1)$ \\
\hline - Radiological diagnosis & $88(41.9)$ & $0(0.0)$ & $0(0.0)$ & $7(30.4)$ & $81(77.9)$ \\
\hline
\end{tabular}

\section{Treatment}

Just under half of patients $(104,49.5 \%)$ received supportive care and did not receive either chemotherapy or radiotherapy. Out of the 106 patients who received active treatment, radiotherapy with concurrent and/or adjuvant chemotherapy was prescribed for $63.2 \%$, while smaller proportions received either radiotherapy or chemotherapy alone (21.7 and $15.1 \%$ respectively). Patients who received supportive care were older $(63.5$ vs $21.7 \%$ aged 75 and over; $\mathrm{p}<0.001)$ and had poorer PS (50.0 vs 8.5\% with PS $3+$; $\mathrm{p}<0.001)$ compared with the patients who received active treatment.

\section{Patients treated with chemotherapy \& radiotherapy}

Of the 67 patients treated with chemo-radiotherapy, 59.7\% received standard radiotherapy, 34.3\% hypofractionated radiotherapy and $6.0 \%$ other radiotherapy regimens (Table 2). In total, 63 patients received concomitant chemotherapy (temozolomide) and four received adjuvant chemotherapy only. Compared with patients who received standard chemoradiation, those who received hypofractionated chemoradiation were older $(27.5$ vs $56.5 \%$ aged 70 and over; $\mathrm{p}=0.022)$ and had poorer PS (13.0 vs $32.5 \%$ with PS $0 ; \mathrm{p}=0.05)$.

High rates of treatment compliance with radiotherapy were observed in both groups. All 23 patients receiving hypofractionated radiotherapy and 37 of the 40 patients receiving standard radiotherapy completed treatment, and the few patients receiving other schedules also completed treatment.

Chemotherapy data are summarized in Table 3. Temozolomide was the only chemotherapy agent received by the study population. Out of the 23 patients receiving hypofractionated chemo-radiotherapy, 21 (91.3\%) received concomitant temozolomide and two received adjuvant chemotherapy only. Ten of the 21 patients who received concomitant temozolomide went on to receive adjuvant chemotherapy. All 40 patients receiving standard chemo- 


\begin{tabular}{|c|c|c|c|}
\hline & $\begin{array}{l}\text { Hypofractionated radiotherapy and } \\
\text { chemotherapy }\end{array}$ & $\begin{array}{l}\text { Standard radiotherapy and } \\
\text { chemotherapy }\end{array}$ & $\begin{array}{l}\text { Other radiotherapy regimen and } \\
\text { chemotherapy }\end{array}$ \\
\hline Numbers & $23(34.3)$ & $40(59.7)$ & $4(6.0)$ \\
\hline Mean age (SD) & $71.0(3.8)$ & $68.8(3.8)$ & $72(4.5)$ \\
\hline \begin{tabular}{|l} 
Age group: \\
$-65-<70$ \\
$-70-<75$ \\
$-75+$
\end{tabular} & $\begin{array}{l}10(43.5) \\
8(34.8) \\
5(21.7)\end{array}$ & $\begin{array}{l}29(72.5) \\
8(20.0) \\
3(7.5)\end{array}$ & $\begin{array}{l}1(25.0) \\
2(50.0) \\
1(25.0)\end{array}$ \\
\hline \multicolumn{4}{|l|}{ Sex: } \\
\hline $\begin{array}{l}\text { - Men } \\
\text { - Women }\end{array}$ & $\begin{array}{l}12(52.2) \\
11(47.8)\end{array}$ & $\begin{array}{l}20(50.0) \\
20(50.0)\end{array}$ & $\begin{array}{l}3(75.0) \\
1(25.0)\end{array}$ \\
\hline \multicolumn{4}{|c|}{ Performance status: } \\
\hline $\begin{array}{l}-0 \\
-1 \\
-2 \\
-3+ \\
- \text { Unknown }\end{array}$ & $\begin{array}{l}3(13.0) \\
14(60.9) \\
3(13.0) \\
2(8.7) \\
1(4.3)\end{array}$ & $\begin{array}{l}13(32.5) \\
16(40.0) \\
5(12.5) \\
2(5.0) \\
4(10.0)\end{array}$ & $\begin{array}{l}1(25.0) \\
1(25.0) \\
1(25.0) \\
0(0) \\
0(0.0)\end{array}$ \\
\hline \multicolumn{4}{|l|}{ Treatment center: } \\
\hline $\begin{array}{l}\text { - Glasgow } \\
\text { - Brighton } \\
\text { - Manchester }\end{array}$ & $\begin{array}{l}9(39.1) \\
2(8.7) \\
12(52.2)\end{array}$ & $\begin{array}{l}7(17.5) \\
7(17.5) \\
26(65.0)\end{array}$ & $\begin{array}{l}0(0.0) \\
0(0.0) \\
4(100.0)\end{array}$ \\
\hline
\end{tabular}

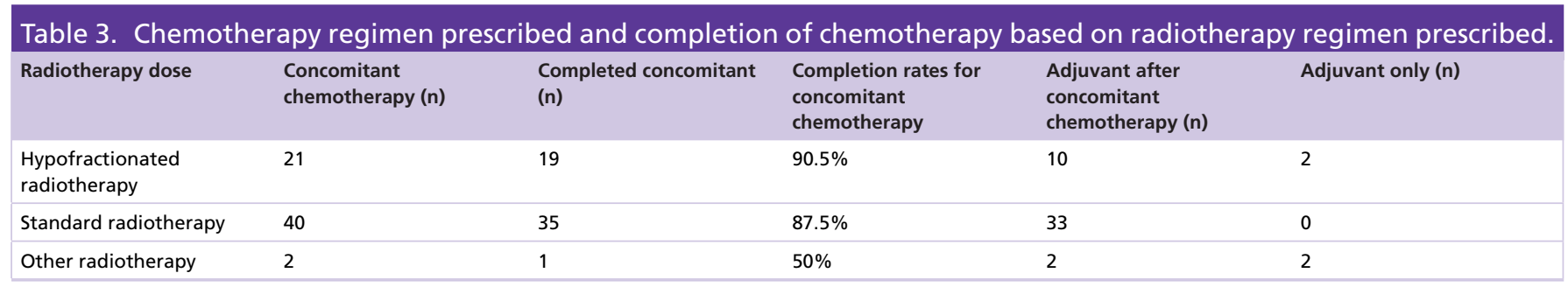

\begin{tabular}{|c|c|c|}
\hline Radiotherapy dose & Frequency & Patients who completed radiotherapy (\%) \\
\hline 40 Gy in 15 fractions & 10 & $80 \%$ \\
\hline 30 Gy in 6 fractions & 11 & $90.9 \%$ \\
\hline 50 Gy in 25 fractions & 1 & $100 \%$ \\
\hline
\end{tabular}

radiotherapy received concomitant chemotherapy. Completion rates for concomitant chemotherapy were similar: $90.5 \%$ in the hypofractionated group and $87.5 \%$ in the standard group $(\mathrm{p}=0.7$ ). Adjuvant therapy was offered to 33 patients after standard treatment. Information on completion of adjuvant chemotherapy was incomplete and therefore not reported.

\section{Patients in other treatment groups}

Sixteen patients received chemotherapy only in the form of temozolomide. Information about dose and number of cycles was incomplete and is therefore not reported.

As shown in Table 4, 23 patients received radiotherapy only. Of these, ten were prescribed 40 Gy in 15 fractions, of whom $80 \%$ completed the course; 11 were prescribed $30 \mathrm{~Gy}$ in six fractions, of whom 91\% completed. One patient prescribed $50 \mathrm{~Gy}$ in 25 fractions completed the course while one patient prescribed 60 Gy in 30 fractions did not. 


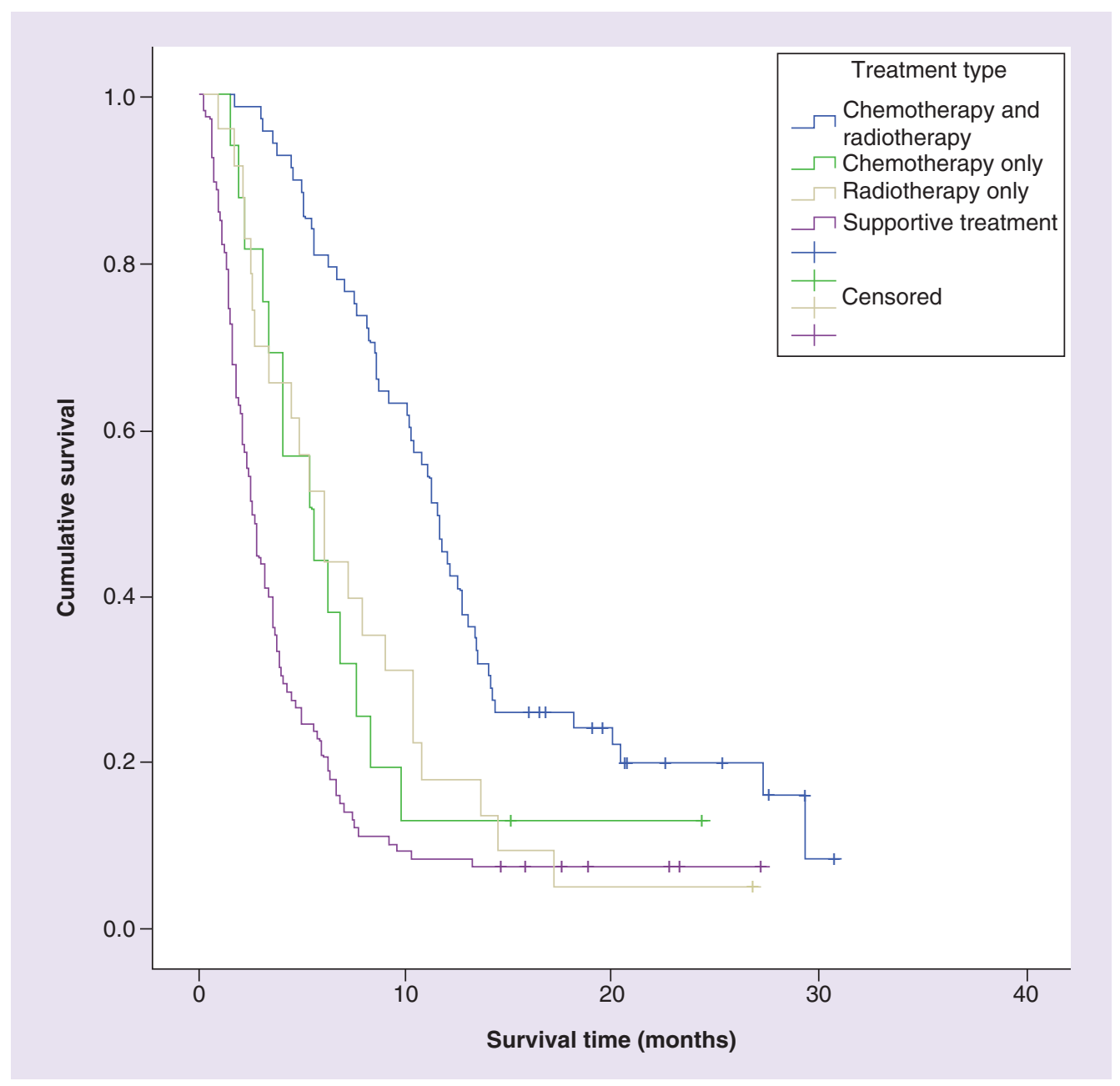

Figure 1. Kaplan-Meier survival curves based on treatment type received.

\section{Survival outcomes}

The median follow-up was 22.6 months, during which 188 patients $(89.5 \%)$ died. Median overall survival for the entire group was 5.0 months (95\% CI: 3.8-6.2 months). Figure 1 shows the survival curves based on treatment received. Patients who received active treatment had a median survival of 9.0 months (95\% CI: 7.6-10.9), whereas patients who received supportive care had a median survival of 2.5 months (95\% CI: 2.1-3.2). Among actively treated patients, those who received chemo-radiotherapy experienced higher overall survival (11.5 months) than those who received either chemotherapy (5.4 months) or radiotherapy (6.0 months) alone ( $\mathrm{p}=0.0005)$.

There was no statistically significant difference in survival by treatment center $(\mathrm{p}=0.75)$, PS $(\mathrm{p}=0.81)$ or $M G M T$ promoter methylation status $(\mathrm{p}=0.41$ ) among actively treated patients (Table 5).

Median overall survival of patients who received standard chemo-radiotherapy was increased by just over 2 months compared with survival among those receiving hypofractionated chemo-radiotherapy (12.5 months [95\% CI: 10.3-14.1] vs 10.2 months [5.0-11.5], respectively, $\mathrm{p}=0.026)$. The corresponding survival curves are shown in Figure 2. Tables $6 \& 7$ show univariate and multivariate adjusted hazard ratios for overall survival, taking account of clinical and treatment characteristics. Hazard of death was increased among patients who received hypofractionated radiotherapy compared with patients who received standard radiotherapy. No other variables showed a statistically significant association with survival in the univariate analysis, although women displayed a reduced hazard compared with men and patients with a PS of 1 or more showed an increase in the hazard of death. Patients receiving hypofractionated radiotherapy continued to show an increased hazard of death in multivariable analyses adjusting for sex, age and PS. The increased hazard persisted when adjustment was made for the propensity of patients to receive hypofractionated radiotherapy. The hazard ratio of death among patients 


\begin{tabular}{|c|c|c|c|c|}
\hline & Number & Median survival (months) & $95 \% \mathrm{Cl}$ & Log rank test $p$-value \\
\hline Actively treated & 106 & 9.0 & $3.8-6.2$ & \\
\hline $\begin{array}{l}\text { Combined chemotherapy and } \\
\text { radiotherapy: }\end{array}$ & 67 & 11.5 & $9.2-12.7$ & 0.0005 \\
\hline $\begin{array}{l}\text { - Hypofractionated } \\
\text { radiotherapy }\end{array}$ & 23 & 10.2 & $5.0-11.5$ & \\
\hline - Standard radiotherapy & 40 & 12.5 & $10.3-14.1$ & \\
\hline - Other radiotherapy & 4 & 5.5 & 5.5 to not reached & \\
\hline Chemotherapy only & 16 & 5.4 & $3.1-7.6$ & \\
\hline Radiotherapy only & 23 & 6.0 & $2.7-9.0$ & \\
\hline Glasgow & 35 & 8.3 & $5.4-10.7$ & 0.75 \\
\hline Brighton & 15 & 10.3 & $6.6-11.1$ & \\
\hline Manchester & 56 & 9.2 & $6.2-12.5$ & \\
\hline PS score $(0-1)$ & 72 & 8.3 & $6.2-10.7$ & 0.82 \\
\hline PS score (2-4) & 27 & 9.8 & $6.0-12.5$ & \\
\hline Methylated MGMT promoter & 21 & 8.6 & $4.1-18.1$ & 0.41 \\
\hline Unmethylated $M G M T$ promoter & 35 & 10.2 & $7.6-11.2$ & \\
\hline
\end{tabular}

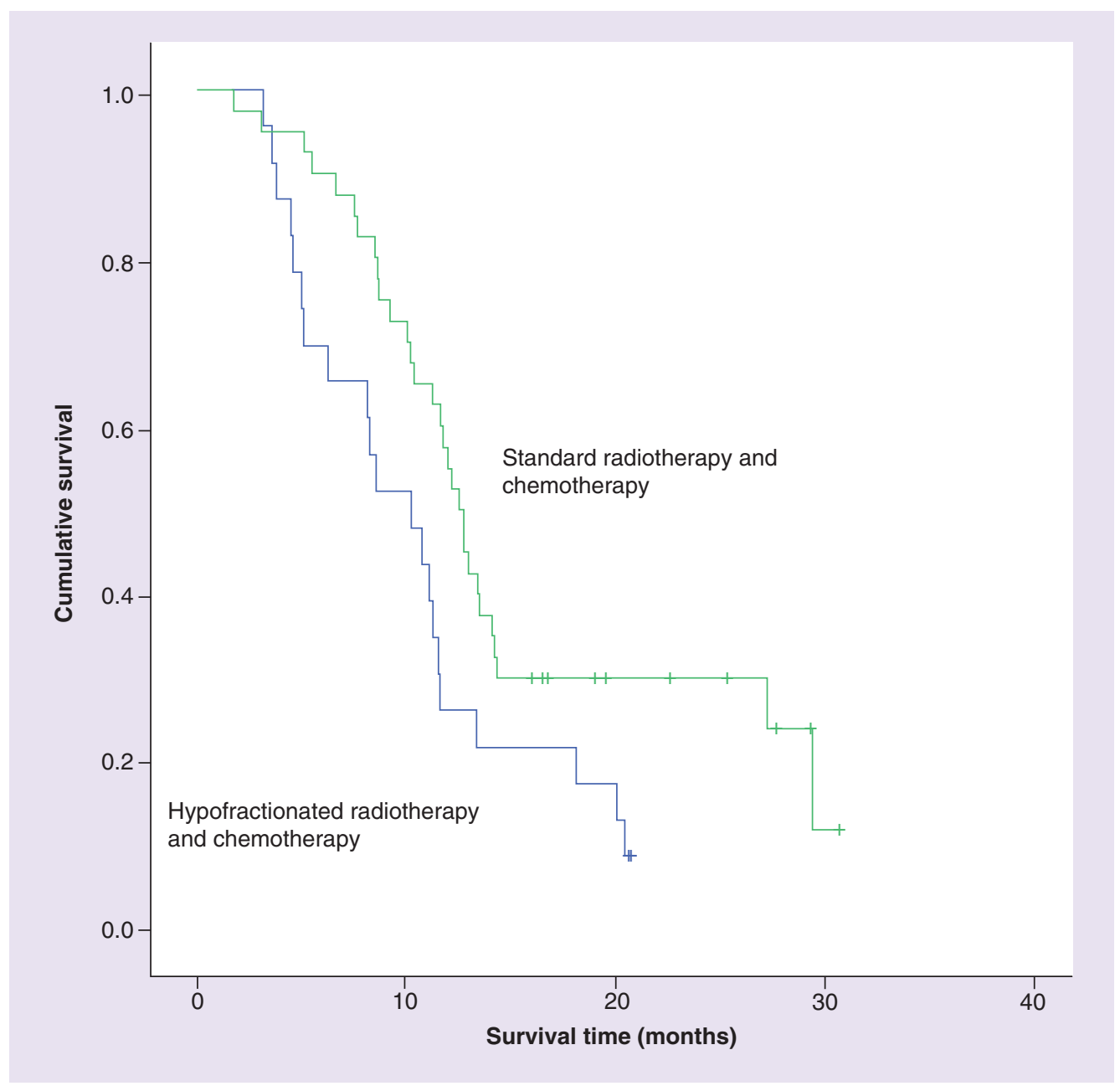

Figure 2. Kaplan-Meier survival curves comparing standard radiotherapy and chemotherapy versus hypofractionated radiotherapy and chemotherapy. 


\begin{tabular}{|c|c|c|c|c|c|c|}
\hline \multirow[t]{2}{*}{ Characteristics } & \multicolumn{3}{|c|}{ Univariable } & \multicolumn{3}{|c|}{ Multivariable } \\
\hline & Hazard ratio & $95 \% \mathrm{Cl}$ & p-value & Hazard ratio & $95 \% \mathrm{Cl}$ & $p$-value \\
\hline \multicolumn{7}{|l|}{ Fractionation: } \\
\hline - Standard & 1 & & & 1 & & \\
\hline - Hypofractionated & 1.89 & $(1.07-3.34)$ & 0.03 & 2.20 & $(1.15-4.21)$ & 0.02 \\
\hline \multicolumn{7}{|l|}{ Sex: } \\
\hline - Men & 1 & & & 1 & & \\
\hline - Women & 0.69 & $(0.39-1.22)$ & 0.21 & 0.59 & $(0.32-1.07)$ & 0.08 \\
\hline \multicolumn{7}{|l|}{ Age group: } \\
\hline$-65-<70$ & 1 & & & 1 & & \\
\hline$-70+$ & 1.01 & $(0.57-1.78)$ & 0.99 & 0.79 & $(0.43-1.44)$ & 0.44 \\
\hline \multicolumn{7}{|l|}{ Performance status: } \\
\hline-0 & 1 & & & 1 & & \\
\hline$-1+$ & 1.26 & $(0.65-2.45)$ & 0.49 & 0.89 & $(0.43-1.84)$ & 0.76 \\
\hline - Unknown & 0.85 & $(0.27-2.66)$ & 0.78 & 0.49 & $(0.14-1.68)$ & 0.26 \\
\hline
\end{tabular}

\begin{tabular}{|c|c|c|c|c|c|c|c|c|c|}
\hline \multirow[t]{2}{*}{ Variables } & \multicolumn{3}{|c|}{$\begin{array}{l}\text { Multivariable adjusting for propensity } \\
\text { score (continuous) }\end{array}$} & \multicolumn{3}{|c|}{$\begin{array}{l}\text { Multivariable adjusting for propensity } \\
\text { score (categorical) }\end{array}$} & \multicolumn{3}{|c|}{$\begin{array}{l}\text { Multivariable stratified by propensity } \\
\text { score category }\end{array}$} \\
\hline & HR & $95 \% \mathrm{Cl}$ & $p$-value & HR & $95 \% \mathrm{Cl}$ & p-value & $H R$ & $95 \% \mathrm{Cl}$ & p-value \\
\hline $\begin{array}{l}\text { Fraction: } \\
\text { - Standard } \\
\text { - Hvpo }\end{array}$ & 1242 & $123-4.78$ & 001 & $\begin{array}{l}1 \\
236\end{array}$ & $1.23-4.52$ & 0.01 & $\begin{array}{l}1 \\
232\end{array}$ & $118-4.56$ & 0.01 \\
\hline Propensity score & 0.39 & $0.09-1.60$ & 0.19 & & & & & & \\
\hline Propensity score: & & & & & & & & & \\
\hline $\begin{array}{l}-<0.25 \\
-0.25-<0.5 \\
-0.5-<0.75 \\
-0.75+\end{array}$ & & & & $\begin{array}{l}1 \\
0.87 \\
0.77 \\
0.36\end{array}$ & $\begin{array}{l}0.32-2.41 \\
0.40-1.47 \\
0.08-1.68\end{array}$ & $\begin{array}{l}0.79 \\
0.43 \\
0.19\end{array}$ & & & \\
\hline
\end{tabular}

receiving hypofractionated radiotherapy compared with standard fractionation ranged from 2.2 to 2.4 depending on the multivariable model employed.

\section{Discussion}

While an increasing body of evidence to guide the management of elderly patients with GBM has been published in recent years, a number of uncertainties remain. Despite Perry et al.'s study [9] showing that addition of TMZ to short course radiotherapy improved survival, no trials have directly compared short course with standard chemoradiotherapy. The main aim of our study was to evaluate the use of hypofractionated chemo-radiotherapy, and to investigate any differences in outcome between elderly GBM patients receiving short course and standard chemoradiation. While the observational and retrospective nature of this study render it susceptible to bias from selection by indication, we reasoned that its findings would be important because a randomized study of standard versus hypofractionated chemoradiation was unlikely to be conducted.

Our study showed that hypofractionated chemo-radiotherapy was delivered to $21.7 \%$ of GBM patients aged 65 or above who received active treatment, and that this regimen yielded similar outcomes to those reported by Perry and colleagues in the recent National Cancer Institute of Canada Clinical Trials Group (NCIC) Elderly Study (median overall survival 10.2 months compared with 9.3 months, respectively) [9]. Despite the publicity surrounding these data, $37.7 \%$ of the actively treated patients in this age group received standard chemo-radiotherapy (60 Gy in 30 fractions). Of note, these patients had a lower risk of death than those receiving hypofractionated chemoradiation. The risk of death continued to be reduced after adjusting for age, sex, PS and the propensity of patients to receive hypofractionated radiotherapy.

It might be reasonable to assume that patients with better prognostic and predictive characteristics would have been offered standard radiotherapy and chemotherapy rather than hypofractionated radiotherapy and chemotherapy by the treating oncologist. Therefore, we would have expected patients who received standard radiotherapy and chemotherapy to have better survival. We tried to account for this by creating a propensity score and included 
this in the survival analysis. A limitation is that there may be unmeasured confounders that influence treatment assignment such as patient preference and clinician decision. The completion rates for adjuvant chemotherapy in patients who received hypofractionated chemo-radiotherapy were incomplete and not considered in the analysis. This may have had a confounding influence on the results observed.

Given the limitations of this retrospective study, we do not claim that these findings advocate against the use of hypofractionated chemo-radiotherapy. Rather they highlight the need for a personalized approach to therapy for elderly GBM patients and, more specifically, the urgent need for validated assessment tools to improve patient and treatment selection.

This study was limited by the relatively small sample size of 210 patients. In particular, subgroup analysis of hypofractionated versus standard chemo-radiotherapy involved only 63 patients. Furthermore, patients were not stratified according to neurosurgical intervention (biopsy, subtotal resection, gross-total resection) because of the small sample size, which may have a confounding effect on the overall survival data. While we were unable to evaluate or compare treatment-related side effects because of variation in data collection across the different centers, we observed no significant difference in treatment completion rates between different radiotherapy or chemo-radiotherapy regimens.

Our decision to include all eligible patients discussed at Neuro-Oncology MDT meetings enabled us to document that half of GBM patients aged 65 or over did not receive any chemotherapy or radiotherapy, and that a significant proportion did not undergo neurosurgical intervention. While these figures appear high, most studies of elderly GBM have excluded patients who have not undergone surgery or received active treatment, so very little data are available for comparison. Our observation that median overall survival for patients receiving no treatment was only 2.5 months is in keeping with the outcome for elderly English patients reported by Brodbelt et al. [4] in 2015, and provides some reassurance that these were appropriate management decisions. While it could be argued that these patients might have experienced longer survival if they had been treated more actively, half of them had very poor PS (ECOG PS $\geq 3$ ) and over $60 \%$ were aged 75 and above. The favorable median survival of 11.5 months in patients receiving chemo-radiation supports the patient selection philosophy of the centers who participated in the study. Our findings indicate that survival data generated by clinical trials are not representative of the elderly GBM population as a whole.

\section{Conclusion}

A large proportion of elderly GBM patients do not receive any oncological treatment. Hypofractionated chemoradiotherapy is being adopted as a treatment regimen in elderly patients with GBM in the UK. Our retrospective study indicated that the patients receiving standard chemo-radiotherapy are at reduced risk of death compared with patients receiving hypofractionated chemo-radiotherapy. While this result might reflect selection of patients with better prognosis to be offered an extended treatment regimen, our analysis did not fully support this interpretation and suggested that standard chemo-radiotherapy for GBM might improve outcomes for some patients. Robust comparison of standard and hypofractionated regimens would require a randomized controlled trial.

\section{Summary points}

- Hypofractionated chemo-radiotherapy is being used in the UK as a treatment regimen in elderly patients with glioblastoma (GBM).

- There was a trend for reduced risk of death for patients who received standard chemoradiation compared with patients who received hypofractionated chemoradiation within the limitations of this retrospective study.

- A large proportion of elderly patients with GBM do not receive definitive treatment.

- Appropriate geriatric assessments are required to allow better assessment of elderly patients with GBM and stratify treatment for these patients.

Financial \& competing interests disclosure

The authors have no relevant affiliations or financial involvement with any organization or entity with a financial interest in or financial conflict with the subject matter or materials discussed in the manuscript. This includes employment, consultancies, honoraria, stock ownership or options, expert testimony, grants or patents received or pending, or royalties.

No writing assistance was utilized in the production of this manuscript. 
Ethical conduct of research

This was an audit which did not require ethical approval based on local policy. Patient data were anonymized and this audit was carried out according to the principles of the Declaration of Helsinki.

\section{Open access}

This work is licensed under the Attribution-NonCommercial-NoDerivatives 4.0 Unported License. To view a copy of this license, visit http://creativecommons.org/licenses/by-nc-nd/4.0/

\section{References}

Papers of special note have been highlighted as: $\bullet$ of interest; $\bullet \bullet$ of considerable interest

1. Arvold ND, Reardon DA. Treatment options and outcomes for glioblastoma in the elderly patient. Clin. Interv. Aging. 9, 357-367 (2014).

2. Dolecek TA, Propp JM, Stroup NE, Kruchko C. CBTRUS statistical report: primary brain and central nervous system tumors diagnosed in the United States in 2005-2009. Neuro Oncol. 14(Suppl. 5), v1-v49 (2012).

3. Morgan ER, Norman A, Laing K, Seal MD. Treatment and outcomes for glioblastoma in elderly compared with non-elderly patients: a population-based study. Curr. Oncol. 24(2), e92-e98 (2017).

4. Brodbelt A, Greenberg D, Winters T et al. Glioblastoma in England: 2007-2011. Eur. J. Cancer 51(4), 533-542 (2015).

5. Stupp R, Mason WP, van den Bent MJ et al. Radiotherapy plus concomitant and adjuvant temozolomide for glioblastoma. N. Engl. J. Med. 352(10), 987-996 (2005).

6. Stupp R, Hegi ME, Mason WP et al. Effects of radiotherapy with concomitant and adjuvant temozolomide versus radiotherapy alone on survival in glioblastoma in a randomised Phase III study: 5-year analysis of the EORTC-NCIC trial. Lancet Oncol. 10(5), 459-466 (2009).

7. Wick W, Platten M, Meisner C et al. Temozolomide chemotherapy alone versus radiotherapy alone for malignant astrocytoma in the elderly: the NOA-08 randomised, Phase 3 trial. Lancet Oncol. 13(7), 707-715 (2012).

8. Roa W, Brasher PMA, Bauman G et al. Abbreviated course of radiation therapy in older patients with glioblastoma multiforme: a prospective randomized clinical trial. J. Clin. Oncol. 22(9), 1583-1588 (2004).

- Important Phase III clinical trial demonstrating short course radiotherapy to be equivalent to extended radiotherapy in terms of overall survival in glioblastoma (GBM) patients aged 60 and above.

9. Perry JR, Laperriere N, O'Callaghan CJ et al. Short-course radiation plus temozolomide in elderly patients with glioblastoma. N. Engl. J. Med. 376(11), 1027-1037 (2017).

-. Landmark Phase III trial demonstrating for the first time that GBM patients aged 65 and above benefit from addition of concomitant and adjuvant temozolomide to short-course radiotherapy. 